\title{
Non-Obstructive Middle Lobe Syndrome: An Unusual Cause of Recurrent Pneumonia in an Elderly Woman
}

\author{
João Rua, Ricardo Marques, Rafael Silva, Bráulio Gomes, Jorge Fortuna \\ Serviço de Medicina Interna B, Centro Hospitalar e Universitário de Coimbra, Coimbra, Portugal
}

Doi: 10.12890/2017_000737- European Journal of Case Reports in Internal Medicine - ㅇ EFIM 2017

Received: 08/09/2017

Accepted: $13 / 11 / 2017$

Published: $23 / 11 / 2017$

How to cite this article: Rua J, Marques R, Silva R, Gomes B, Fortuna J. Non-obstructive middle lobe syndrome: an unusual cause of recurrent pneumonia in an elderly woman. EJCRIM 2017;4: doi:10.12890/2017_000737.

Conflicts of Interests: The Authors declare that there are no competing interests.

This article is licensed under a Commons Attribution Non-Commercial 4.0 License

\section{ABSTRACT}

An 84-year-old woman had five episodes of pneumonia in 4 months. Despite extensive comorbidities and advanced age, her health status was good and the recurrence seemed unjustified. Exhaustive background investigation revealed 14 episodes of right-sided pneumonias during the 3 previous years and an inconclusive investigation with CT of the thorax and bronchofibroscopy, despite some fibrotic and atelectasic alterations in the right middle lobe. A new right-sided x-ray showed a wedge-shaped density extending anteriorly and inferiorly from the hilum, and CT of the thorax revealed aggravated middle lobe abnormalities with thickening of the bronchial wall and segment atelectasis, without any visible airway obstruction. After extensive work-up had excluded other causes of recurrent pneumonia and immunodeficiency, a non-obstructive middle lobe syndrome (MLS) was deemed responsible for the repeated episodes of pneumonia. MLS is characterised by chronic hypoventilation and atelectasis of the middle lobe, facilitating secretion accumulation, chronic inflammation and repeated infection. After treatment with bronchodilators and immunostimulants was initiated, the patient experienced no recurrences for several months.

\section{LEARNING POINTS}

- Recurrent pneumonia is common and has several possible causes. Investigation should be directed by whether or not the location of the episodes varies, suggesting a systemic condition or a local abnormality, respectively.

- Middle lobe syndrome (MLS) is characterised by chronic hypoventilation of the right middle lobe resulting in atelectasis and secretion accumulation, sometimes causing recurrent pneumonia, and should therefore be considered in the differential diagnosis.

- MLS is not unusual in children with asthma but is quite uncommon in the elderly despite age-related changes in the characteristics of airways with reduced elastic recoil and mucociliary clearance.

\section{KEYWORDS}

Recurrent pneumonia, middle lobe syndrome, middle lobe atelectasis

\section{INTRODUCTION}

Middle lobe syndrome (MLS) is characterised by chronic hypoventilation of the right middle lobe (RML) or lingula lobe, resulting in atelectasis, secretion accumulation, chronic inflammation, respiratory symptoms and recurrent infection ${ }^{[1]}$. The anatomy of the lobar bronchi favours transient hypoventilation due to inflammation or obstruction. It is relatively frequent in children with asthma but uncommon in older adults $^{[2,3]}$. We present a case of recurrent pneumonia and describe the diagnostic pathway leading to the diagnosis of non-obstructive MLS. 


\section{CLINICAL CASE}

An 84-year-old Caucasian woman was admitted to the Internal Medicine department due to pneumonia. She recovered well but had apparent bacteraemia the night before her planned discharge, prompting new blood tests, urinalysis and an x-ray of the thorax. All results were normal and the patient was released. However, she returned 5 days later in respiratory failure with acute pulmonary oedema and extensive right-sided pneumonia. This was the fifth episode in the last 4 months.

The patient had considerable comorbidities including heart failure due to ischaemic cardiomyopathy, arterial hypertension, type 2 diabetes mellitus and chronic kidney disease. Nonetheless, her clinical status was good, so this raised some red flags and an extensive background investigation was conducted and showed 14 episodes of pneumonia over the previous 3 years. The patient had already undergone CT of the thorax and bronchofibroscopy 18 month previously. The first showed atelectasis of the lateral segment of the RML with irregularity of the corresponding bronchi without any occluding formation, while the latter provided no relevant information as biopsies, bronchial lavage, aspirate and microbiology were all normal/negative. The lung collapse was deemed passive and the patient was referred for short-term respiratory kinesiotherapy. However, she continued to worsen, with progressively shorter intervals between episodes.

Imaging was repeated. A right-sided thoracic x-ray showed a wedge-shaped density extending anteriorly and inferiorly from the hilum, while CT of the thorax showed exposed thickening of the RML bronchi with discrete bronchiectasis and considerable atelectasis of the lobe. An echocardiogram showed no signs of endocarditis (Fig. 1 and 2). Known causes of chronic pneumonia were excluded by serial blood cultures for mycobacteria and fungi[ ${ }^{[4,5]}$, and serology for Coxiella burnetii, Mycoplasma pneumoniae, Legionella pneumophila, Chlamydophila pneumoniae and Mycobacterium spp.HIV infection, autoimmune diseases, complement and immunoglobulin deficiencies were also excluded ${ }^{[6]}$.

In light of the recurrent pneumonia, the first step was to determine if repeated infections were due to immunodeficiency, chronic infection or morphological abnormality. The recurrent location suggested the latter, as did the examinations showing persistent atelectasis of the RML, consistent with MLS, and the exclusion of common chronic infections and immunodeficiencies. The second phase was to determine the type of MLS and search for a treatable cause ${ }^{[4,5,7]}$. The investigations did not suggest an obstruction in accordance with the previous bronchofibroscopy. A new bronchofibroscopy was deemed unnecessary given the short amount of time that had passed and the previous unremarkable findings.

Non-obstructive MLS was diagnosed and bronchodilators, mucolytics and immunostimulants were initiated, with good tolerability. The patient had no infectious episodes for 6 months, until her death from unrelated causes. Despite her short survival, the treatment considerably improved her quality of life.

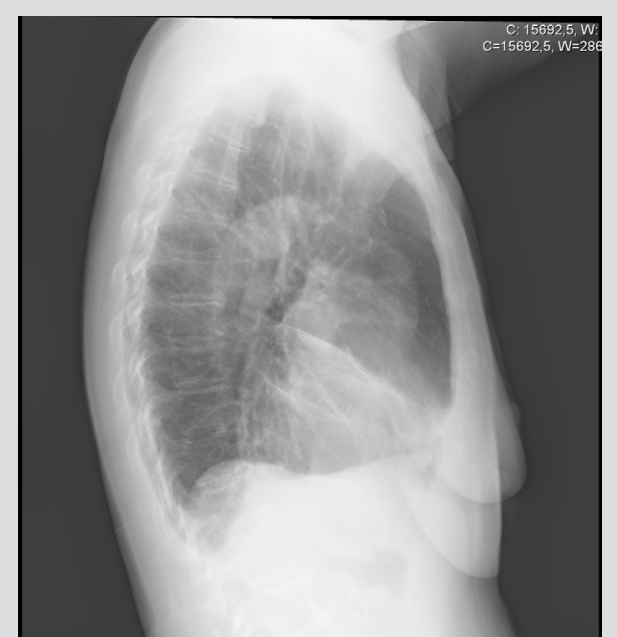

Figure 1. Right sided thorax $x$-ray showing the typical wedge shaped density extending anteriorly and inferiorly from the hilum

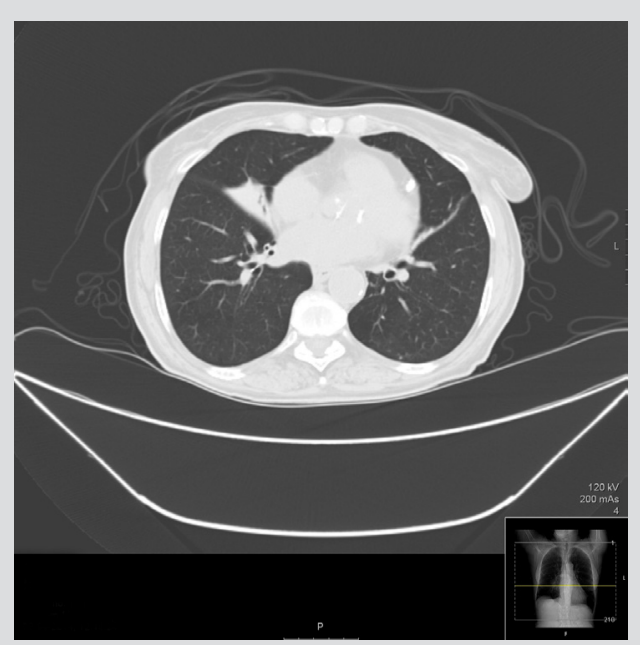

Figure 2. CT of the thorax showing middle lobe atelectasis and bronchiectasis

\section{DISCUSSION}

Investigation of recurrent pneumonia is complex and often troublesome. The starting point should be to determine if the locations of the different episodes of pneumonia differ. If the sites are the same, a localized morphological abnormality or unresolved local chronic infection is likely, making imaging such as CT of the thorax and bronchofibroscopy a priority, as they can clarify airway patency and reveal 
an obstruction. Bronchofibroscopy allows evaluation of small lesions invisible on CT. Biopsies should be taken of abnormal masses and specimens collected for pathogen identification. Localized chronic infections due to Mycobacterium, Coxiella, Mycoplasma, Actinobacteria and fungi such as Aspergillus and Histoplasma, should always be excluded. On the other hand, different locations suggest a systemic condition that weakens the patient, for instance immunodeficiency or a global ventilation disturbance such as asthma. Consideration must be given to chronic immunosuppressive medication. HIV, complement and immunoglobulin deficiencies should be excluded and the effect of diabetes, neoplasia or autoimmune diseases must be taken into account ${ }^{[8-11]}$.

In our case, as the location was always the middle lobe, morphological abnormality was suspected. The CT of the thorax and bronchofibroscopy confirmed the chronic hypoventilation and atelectasis of the middle lobe and some discrete bronchiectasis but revealed no obstructions. As all causes of chronic pulmonary infection and immunodeficiency were excluded, a diagnosis of non-obstructive MLS was made. An intricate interaction between the diabetes immunosuppression, bronchiectasis and the known alterations in RML anatomy with age-induced airway changes such as decreased lung and thoracic compliance, diminished respiratory muscle strength and impaired mucociliary clearance, was considered to have caused pneumonia recurrence late in our patient's life ${ }^{[12]}$.

MLS was first described in 1948 by Graham et al. ${ }^{[3]}$ as a chronic or recurrent collapse of the middle lobe due to hypoventilation resulting from mechanical obstruction or transient mucosal inflammation ${ }^{[2]}$. Limited collateral ventilation and the specific anatomy of the bronchus, which is narrow, long and has an acute take-off angle, are physiopathologically decisive ${ }^{[1,7,13]}$. There are two types of MLS, obstructive and non-obstructive, with the first subdivided into intrinsic or extrinsic, according to the nature of the obstruction ${ }^{[2]}$. Tumours and enlargement of peribronchial lymph nodes are the main causes of obstructive MLS, with transient inflammation and bronchial hyper-responsiveness causing non-obstructive MLS ${ }^{[1]}$.

The most frequent clinical features are recurrent cough, dyspnoea, wheezing, chills, fever, haemoptysis and pneumonia ${ }^{[2,13-15]}$. A typical wedge-shaped density extending antero-inferiorly from the hilum in the lateral thoracic $x$-ray suggests the diagnosis ${ }^{[1]}$. CT of the thorax and bronchofibroscopy confirm the atelectasis of the middle lobe and are used to determine if there is an obstruction, clarifying the type of MLS and the corresponding aetiology ${ }^{[1,14]}$.

Treatment is linked to the type of MLS. If obstructive, surgery is needed to remove the cause or the entire lobe. In non-obstructive MLS, a medical approach is preferred, with bronchodilator and mucolytic agents, respiratory kinesiotherapy and immunostimulants ${ }^{[1,2,16-18]}$.

MLS is an unusual condition without a defined frequency in the elderly population ${ }^{[1]}$. Its physiopathology and the effects of ageing on the respiratory system ${ }^{[12]}$ suggest it is significantly underdiagnosed in this population.

\section{REFERENCES}

1. Gudbjartsson T, Gudmundsson G. Middle lobe syndrome: a review of clinicopathological features, diagnosis and treatment. Respiration 2012;84:80-86.

2. Eid NS, Eckerle M. Right middle lobe syndrome. Medscape.com, April 2015.

3. Graham EA, Burford TH, Mayer JH. Middle lobe syndrome. Postgrad Med 1948;4:29-34.

4. Shah A, Behera S, Panjabi C. Middle lobe syndrome: a rare presentation of allergic bronchopulmonary aspergillosis. Eur Ann Allergy Clin Immunol 2014;46:147-151.

5. Kala J, Sahay S, Shah A. Bronchial anthracofibrosis and tuberculosis presenting as a middle lobe syndrome. Primary Care Resp J 2008;17:51-55.

6. Chen HA, Lai SL, Kwang WK, et al. Middle lobe syndrome as the pulmonary manifestation of primary Sjögren's syndrome. Med J Aust 2006;184:294-295.

7. Hamad AM, Elmistekawy E, Elatafy E. Chronic atelectasis of the left lower lobe: a clinicopathological condition equivalent to middle lobe syndrome. Interact Cardiovasc Thorac Surg 2012;15:618-621.

8. Dang TT, Majumdar SR, Marrie TJ, Eurich DT. Recurrent pneumonia: a review with focus on clinical epidemiology and modifiable risk factors in elderly patients. Drugs Aging 2015;32:13-19.

9. Stilwell P. Recurrent pneumonias. Clinicaladvisor.com, 2016, http://www.clinicaladvisor.com/hospital-medicine/recurrent-pneumonias/article/602740/. [Accessed on 10/09/17]

10. Ost D, Fein A, Feinsilver SH. Nonresolving pneumonia. uptodate.com, 2017.

11. Rashid A, Nanjappa S, Greene JN. Infectious causes of right middle lobe syndrome. Cancer Control 2017;24:60-65.

12. Janssens JP, Krause KH. Pneumonia in the very old. Lancet Infect Dis 2004;4:112-124.

13. Banyai AL. The middle lobe syndrome and its quasi variants. Chest 1974;65:135.

14. Mejía Lozano P, Pérez Ortiz E, Puchaes Manchón C. Middle lobe syndrome. Emergencias 2009;21:475.

15. Shaikhrezai K, Khorsandi M, Zamvar V. Middle lobe syndrome associated with major haemoptysis. J Cardiothorac Surg $2013 ; 8: 84$.

16. Blaivas A, Strauss W. Middle lobe syndrome in the left lower lobe in chronic obstructive pulmonary disease. Primary Care Resp J 2009;18:331-333.

17. Meteroğlu F, Şahin A, Eren TS. Middle lobe syndrome: a retrospective analysis. Türk Gögüus Kalp Damar Cerrahisi Dergisi 2013;21:718-722.

18. Pejhan S, Salehi F, Niusha S, et al. Ten years' experience in surgical treatment of right middle lobe syndrome. Ann Thorac Cardiovasc Surg 2015;21:354-358. 